\title{
A Prediction Method to Improve Training Management
}

\author{
Mohamed Elglaad \\ Al-Azhar ElSharief \\ Damietta, Egypt
}

\author{
A. A. Ewees \\ Computer Teacher Prep. Dept. \\ Damietta University \\ Damietta, Egypt
}

\author{
E. E. Abd-Elrazek \\ Computer Teacher Prep. Dept. \\ Damietta University \\ Damietta, Egypt
}

\begin{abstract}
The current research aims to use expert systems techniques to predict the training needs of trainees based on several factors related to the functional status of each employee (group, quality, job, training courses), which are essential factors in this forecasting process; because of the diversity of the training needs in light of the job conditions, technological and international development. So, the hold makers are imposed to identify these needs which are determined as the most important processes lead to success the training process. In this paper, three prediction algorithms were used: Bagging, NaviaBayes, and Neural Network to predict the training needs of the trainees in order to support and decision-making among the decision makers in education and increase the accuracy as well as the effectiveness of the training courses. The dataset consisted of 334 cases. The results of the experiments showed that the Bagging algorithm achieved the better accuracy against the rest of the algorithms.
\end{abstract}

\section{Keywords}

Bagging; NaviaBayes; Neural Network; Predicting Training Needs.

\section{INTRODUCTION}

The analysis of employees' data has a great importance in scientific and technological processes in general, as well as, at the quantitative and qualitative development of the educational institutions and its administrative systems in particular. So, this paper has been focused on the training needs of employees in order to keep up with the progress of scientific, technological and administrative progress. On training needs that are in line with the latest developments and technological innovations. Therefore, we need to develop training and support trainers and trainees not only to acquire knowledge but also to develop the skills necessary for them by consolidating the concept of learning and training for life. Training is an essential function of human resources management; training refers to change, and improvement and development. It means change for the better, or development of the person in his knowledge, trends, abilities, skills, and ideas. Ultimately improving his performance and thus increasing his productivity [1]. Computer and internet-based learning and training systems in recent years have developed significantly following the introduction of expert system technology. This technology has added a new dimension to learning, given it roles and activities that never existed before, and has given a great role to the training process because of it a system that analyzes the needs and specialization of each person individually. Consequently, takes into consideration the individual differences between employees who need training according to their data record [2].

When we look at the mechanism for trainees' distribution in various training programs, it was observed that the trainees were selected randomly without taking into account previous courses, specific group, or job.

This paper has handled the previous problem through an expert system to predict the training needs of the trainees to help and support the decision-making process. This paper is organized as following: Section 2 displays previous studies, Section 3 reviews the Bagging algorithm, Section 4 reviews the NaviaBayes algorithm, Section 5 reviews the Neural Network algorithm, Section 6 discusses the experiment and results, Section 7 displays the conclusion and future works.

\section{LITERATURE REVIEW}

The training needs are changing in light of the international changes, So, authorities should identify employees training needs which are considered one of the most important processes that lead to the training success. From [3] the training needs can be achieved through the establishment of a special training unit in each organization to be responsible for identifying staff training needs and follow they up at future. That agrees with the goals of the current paper which estimate training needs for the trainees but by proposing an electronic expert system to predict the training needs of the organization in various branches. These studies [4] and [5] adopted information technology in management, training, and evaluation; and recommended to establish a national technological base in the training service.

Siraj [5] recommended that there is a necessity for the presence of a written, systematic and integrated plan to identify training needs. The need to give the process of identifying the training needs of enough time and funding required for it to be accomplished perfectly, and to outreach workers of the importance of the process of identifying the training needs for them. The [6] concluded to numbers of results; the most important one is: identification of training needs as a necessary step in the planning of the training process. It also contributes in the rationalization of trainingmaking industry.

A study [7] recommended the introduction of expert systems in decision support operations, which is confirmed by [8] Also, [2] recommended holding different workshops in all research, scientific institutions, and colleges to raise awareness of the role of systems expert in decision-making in training.

In [9] the result showed that the neural network algorism has high performance rather than another algorism; in addition, [10] suggested to use $\mathrm{C} 4.5$ for prediction training needs.

\section{PREDICTION METHODS 3.1 Bagging}

Bagging predictors is a way to generate many predictive versions and use them to get grouped predictors. The average assembly across versions is expected during the numerical 
result and the multiplicity of the vote when predicting class. Multiple versions are formed by repeating bootstrap replicates of the learning set and using these groups to learn as new learning groups [11]. The following algorithm shows the sequence of this algorithm.

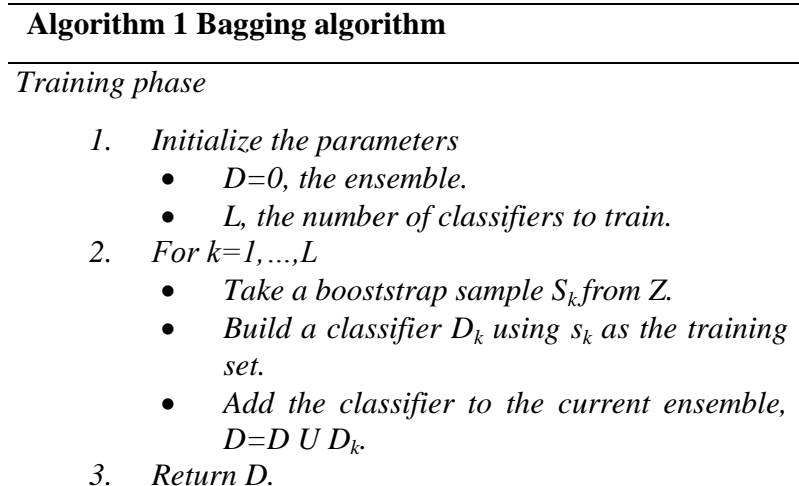

Classification phase

4. Run $D_{l}, \ldots ., D_{l}$ on the input $x$.

5. The class with the maximum number of votes is chosen as the label for $x$.

\subsection{NaviaBayes}

The Naive Bayes algorithm is a simple probabilistic classifier that calculates a set of probabilities by counting the frequency and combinations of values in a given data set. The algorithm uses Bayes theorem and assumes all attributes to be independent given the value of the class variable. This conditional independence assumption rarely holds true in real world applications, hence the characterization as Naive yet the algorithm tends to perform well and learn rapidly in various supervised classification problems [12].

NaviaBayes classifier is based on Bayes' theorem and the theorem of total probability. The probability that a document with vector $\mathrm{x}=\left\langle x_{1}, \ldots, x_{n}\right\rangle$ belongs to hypothesis $\mathrm{h}$ is [13] [14]

$$
P(h 1 \mid x i)=\frac{P x i \mid h 1 P(h 1)}{P x i \mid h 1 P h 1+P(x i \mid h 2) P(h 2)}
$$

Here, $P(h l \mid x i)$ is posterior probability, while $P(h l)$ is the prior probability associated with hypothesis $h 1$.

Form different hypotheses, we have:

$$
P(x \mid)=\sum_{j=1}^{n} P(x i \mid h j) P(h j)
$$

Thus, we have

$$
P(h 1 \mid x i)=\frac{P(x i \mid h 1) P(h 1)}{P(x 1)}
$$

\subsection{Neural Network}

Neural Networks are composed of simple elements operating in parallel. These elements are inspired by biological nervous systems. As in nature, the connections between elements largely determine the network function. A neural network can be trained to perform a particular function by adjusting the values of the connections (weights) between elements. Neural networks have been trained to perform complex functions in various fields, including pattern recognition, classification, identification, vision, speech, and control systems [15].

MLP is a feed forward neural networks model, which is a set of input data to be on the set of the appropriate output. MLP consists of multiple layers of the nodes in the Directed Graph, with each layer fully connected to the next stage. Except input node (input nods) each node is a nerve cell or a processing element with the (function) nonlinear activation [16]. MLP uses a technique called supervisory learning back propagation to train networks [17]. MLP is a modification of criteria Liner Perceptron; it can be distinguished between data that cannot be separated linearly [18]

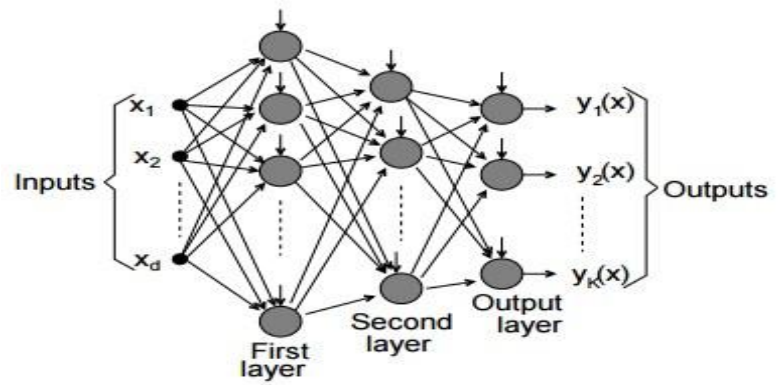

Fig. 1: Multi-Layer Perception

The output of the MLP is calculated as follows [19]:

- The weighted sums of inputs are first calculated by:

$$
s_{j}=\sum_{i 1}^{n}\left(W_{i j} . X_{i}\right)-\theta_{j}, j=1,2, \ldots h
$$

where $n$ is the number of the input nodes, Wij shows the connection weight from the ith node in the input layer to the $j$ th node in the hidden layer, $\theta j$ is the bias (threshold) of the $j$ th hidden node, and $X i$ indicates the $i$ th input.

- The output of each hidden node is calculated as follows:

$$
S_{j}=\operatorname{sigmoid}\left(s_{j}\right)=\frac{1}{\left(1+\exp \left(-s_{j}\right)\right)}, j=1,2, \ldots, h
$$

The final outputs are defined based on the calculated outputs of the hidden nodes as follows:

$$
\begin{aligned}
& o_{k}=\sum_{j=1}^{h}\left(w_{j k} S_{j}\right)-\theta_{k}^{h}, k=1,2, \ldots, m \\
& O_{k}=\operatorname{sigmoid}\left(o_{k}\right)=\frac{1}{\left(1+\exp \left(-o_{k}\right)\right)}, k=1,2, \ldots h
\end{aligned}
$$

where $w_{\mathrm{jk}}$ is the connection weight from the $j$ th hidden node to the $k$ th output node, and $O_{k}$ is the bias (threshold) of the $k$ th output node. The most important parts of MLPs are the connection weights and biases. As seen in the equations, the weights and biases determine the final values of output. Training an MLP involves finding optimum values for weights and biases in order to achieve desirable outputs from certain given inputs.

\section{EXPERIMENTS AND RESULTS}

\subsection{Dataset specification}

Dealing with trainee record (government employee), a lot of jobs information on a trainee record are found. In this paper, some data has been taken and handled to create the experimental dataset.

\subsubsection{Data Source}

The database prepared by the researchers, which includes 4731 records in 25 tables, each table includes many of the properties (fields), each property (field) contains sub-properties that used to predict training needs.

\subsubsection{Data Specialize}

The following section includes a description of the data that have been obtained from the databases of trainees to build the dataset; The following features have been selected manually to be used in this paper. 


\section{A. Employment status data}

This data is linked to the functional status of the trainee, which consists of their specific groups and jobs.

A specific group: is a group appointed by administrative. They are six groups (finance - accounting - administrative development - written Jobs - social services - nutrition and agriculture - documents and libraries), each one is different from the other.

Jobs: it is the function that occupied by the trainee. It is noted that each function was included into the quality of the group as in Table (1).

Table 1. Specific group

\begin{tabular}{|c|c|}
\hline Specific group & Jobs \\
\hline \multirow{3}{*}{$\begin{array}{l}\text { Finance and } \\
\text { accounting: }\end{array}$} & Financial Specialist \\
\hline & Procurement, stores specialist \\
\hline & Specialist financial inspection \\
\hline \multirow{3}{*}{$\begin{array}{l}\text { Administrative } \\
\text { development: }\end{array}$} & Specialist Affairs Employees \\
\hline & Specialist administrative inspection \\
\hline & Administrative affairs \\
\hline \multirow{2}{*}{$\begin{array}{l}\text { Functions } \\
\text { written: }\end{array}$} & Starter affairs institutes \\
\hline & Starter affairs area \\
\hline \multirow{2}{*}{ Social services: } & A social worker \\
\hline & A specialist sports \\
\hline \multirow{2}{*}{$\begin{array}{l}\text { Nutrition and } \\
\text { agriculture: }\end{array}$} & Nutrition supervisor \\
\hline & Nutrition specialist \\
\hline $\begin{array}{l}\text { Documents and } \\
\text { libraries: }\end{array}$ & Specialist documents and libraries. \\
\hline
\end{tabular}

\section{$B$. Training courses}

The database has a table that contains computer courses of obtained data. Computer courses are (Windows - Word - Excel - Access - Internet). At this stage, the querying of desired characteristics of the selected tables from the database. The table staff was using "specific group" field where the field is located on any quality specific group appointed by the employee whether teaching, inviting, or administrative development funding, or accounting \& succession, and so on. The position field, which determines the position of each according to the quality group designated by the employee.

The courses were filtered from obtained training courses. Since the current research focused on computer courses for administrator workers. Namely, a Windows operating system, Word, Excel, Access databases program and Internet.

\subsubsection{Data preparation}

At this stage, dataset has been established in according to selected properties with various examples for use. The data set experiment was established through previous data processing from the database to be introduced to Weka program as inputs into the following form:

Table 2. The dataset information

\begin{tabular}{|c|c|c|}
\hline Relation & Instances & Attributes \\
\hline Dataset Training & 334 & 7 \\
\hline
\end{tabular}

According to Table 2, Relation means: Dataset Training Name, Instance means the number of samples: 334, Attributes means the number of features: 7 (Specific group - Windows Jobs-Internet - Word - Excel - Access).

\subsection{Experiment and Discussion}

The experiment was done by preparation of processing dataset, which was drawn from the data of trainees.

The training set includes seven attributes: Specific group, Jops, Word, Windows, Excel, Access, and Internet, in addition with the class. After that, the learned classifiers are tested using 10 cross-validations. The value that has the maximum number of correctly classified instances is kept as the optimal one Different trials have been done to determine the effectiveness of each algorithm separately to get the best results. Therefore, the best performance among those algorithms can be recorded; were taking the average results for each algorithm as Table 3 . The parameters of MLP algorithm were as follows: Hidden layer equals 3, which is a constant value at the level of testing. In Bagging algorithm number of iterations variable equals 2 .

The mean absolute error (MAE) and accuracy are used to evaluate the results [20] as in Equations (7) and (8) [21] respectively.

$$
\text { MAE }=\frac{1}{n} \sum_{i=1}^{n}\left|f_{i}-y_{i}\right|
$$

where, $f_{i}$ is a predicted value and $y_{i}$ is the true value.

$$
\text { Accuracy }=\frac{T P+T N}{T P+T N+F P+F N} \times 100
$$

where, TP is the True Positives, FP is the False Positives, and $F N$ is the False Negative.

Table 3. The MAE and accuracy results of all algorithms in the experiment.

\begin{tabular}{|c|c|c|c|}
\hline Algorithm & MAE & Accuracy & $\begin{array}{c}\text { Incorrectly } \\
\text { Classified Instances }\end{array}$ \\
\hline Bagging & 0.0310 & $94 \%$ & $6 \%$ \\
\hline NaiveBayes & 0.0465 & $92 \%$ & $8 \%$ \\
\hline MLP & 0.0698 & $86 \%$ & $14 \%$ \\
\hline
\end{tabular}

It can be observed from Table 3 that, the results of the average of experiments indicate that the Bagging achieved better results in all measure; followed by NaiveBayes which achieved good result in accuracy (Correctly Classified Instances) is same Bagging algorithm but the result of MAE with high then Bagging; followed by MLP accuracy less than another algorithm.

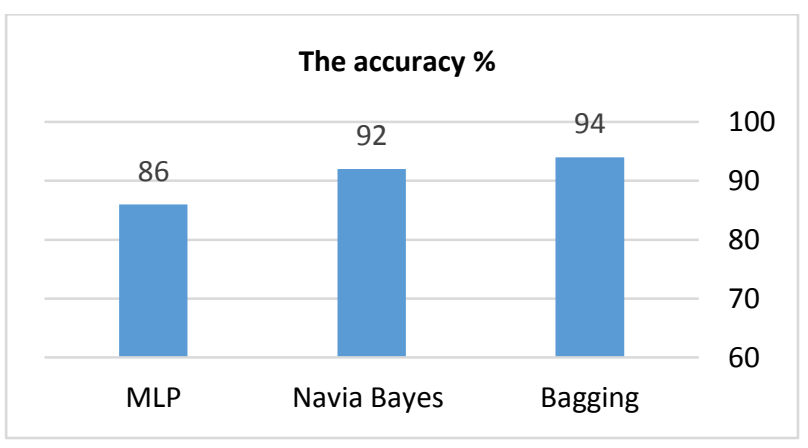

Fig 2: The accuracy of the experiments results 


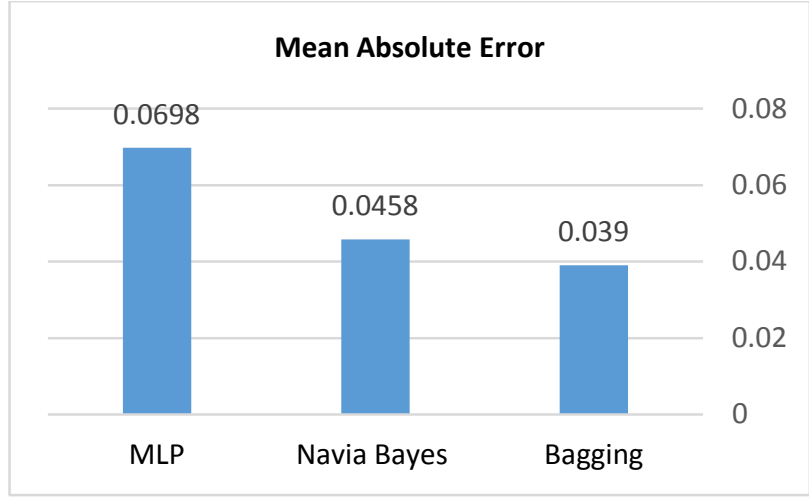

Fig 3: The MAE values of the experiments

It can be seen from Figures 2 and 3 that, the best results is for the Bagging algorithm; this is due to their high expectation in training needs, its accuracy reached $94 \%$ and the MAE is 0.039 .

Table 4 shows a comparison between human expert and the predicted results of the three algorithms (50 results as a test set); since, Table 4 shows the results of the three prediction algorithms compared with the predicted results of the human expert on a sample of trainees

Table 4. A sample of the predicted results of all algorithms

\begin{tabular}{|c|c|c|c|c|}
\hline ID & $\begin{array}{c}\text { Human } \\
\text { Expert }\end{array}$ & MLP & NaviaBayes & Bagging \\
\hline 1 & 3 & 3 & 4 & 4 \\
\hline 2 & 3 & 5 & 3 & 3 \\
\hline 3 & 3 & 3 & 3 & 3 \\
\hline 4 & 3 & 4 & 4 & 4 \\
\hline 5 & 3 & 3 & 3 & 3 \\
\hline 6 & 4 & 4 & 4 & 4 \\
\hline 7 & 4 & 4 & 4 & 4 \\
\hline 8 & 4 & 4 & 4 & 4 \\
\hline 9 & 5 & 3 & 3 & 3 \\
\hline 10 & 5 & 5 & 5 & 5 \\
\hline 11 & 4 & 1 & 4 & 4 \\
\hline 12 & 1 & 3 & 1 & 1 \\
\hline 13 & 4 & 1 & 4 & 4 \\
\hline 14 & 4 & 3 & 4 & 4 \\
\hline 15 & 4 & 4 & 4 & 4 \\
\hline 16 & 4 & 3 & 4 & 4 \\
\hline 17 & 2 & 2 & 2 & 2 \\
\hline 18 & 4 & 4 & 4 & 4 \\
\hline 19 & 2 & 2 & 2 & 2 \\
\hline 20 & 2 & 2 & 2 & 2 \\
\hline 21 & 4 & 4 & 4 & 4 \\
\hline 22 & 4 & 4 & 4 & 4 \\
\hline 23 & 5 & 5 & 5 & 5 \\
\hline 24 & 4 & 4 & 4 & 4 \\
\hline 25 & 4 & 4 & 4 & 4 \\
\hline
\end{tabular}

From above table, the best algorithm for predicting was Bagging followed by NaviaBayes.

\section{CONCLUSION AND FUTURE WORK}

The success of the training courses depends mainly on the training needs of the trainees. The process of identifying these needs is rather complex. Therefore, the use of artificial intelligence algorithms is useful in reaching as an expert system to support and take decisions at different stages of training. From the traditional mode of management to an electronic system capable of predicting training needs away from personal opinions and the mood of the decision-maker. The current paper based on the use of artificial intelligence algorithms to predict these needs; the MLP, Bagging, and NaviaBayes algorithm was used to perform this task, which was chosen to determine the most accurate and effective courses. The experimental results proved that the Bagging algorithm is the more suitable for this task. This is due to its high predictability training with accuracy equals $94 \%$ and MAE equals 0.039 , while NaivaBayes algorithm has less accuracy ratio, it reached $92 \%$ and high MAE rather than Bagging; whereas, the MLP algorithm came in the last rank with accuracy equals $86 \%$. So, this paper recommends Bagging algorithm for using to predict employees training needs. In future, an attempt will be made to expand training areas to ensure system quality and effectiveness.

\section{REFERENCES}

[1] Bahrani, M. (2014). Training Needs of Faculty Members in Applied Sciences Colleges in the Sultanate of Oman, not published, Faculty of Science and Arts, University of Nizwa

[2] Kfoury, Mohamed Ahmed Kamal Ibrahim. (2015). Construction of a proposed expert to specialist educational technology system in the light of professional competencies, requirements and functional effectiveness in meeting the future training needs. Ph.D thesis, not published, Faculty of Education, Helwan University.

[3] Ghuraibi, Afaf Abdullah. (2009). Development of a training system during the service in the light of technological development requirements: prospective study. Ph.D thesis, not published, Faculty of Education in Damietta, Mansoura University.

[4] Qouta, Marwa (2008). Teacher in-service training in Damietta in the light of the requirements of the era of globalization. Master thesis, not published, Faculty of Education, Damietta University.

[5] Siraj, Ragab Abdullah (2010), The reality of the process of determining for workers in nongovernmental organizations training needs, Master thesis, Azhar Gaza University, Faculty of Economics and Administrative Sciences.

[6] Troanh, Tahseen Ahmed. (2011). Identify training needs as a basis for the planning process in the security services. Seminar modern methods of planning and training (both theory and practical) in the security services. Graduate School, University of Nayef.

[7] Ahmed, Mohammad Mohammadi Hassania. (2009). Establishment a system to help students choose the physical components harmonized to assemble the computer. Master thesis, not published, Faculty of Qualitative Education, Mansoura University.

[8] Abdullah, Khababah and Abdul Wahab, Jabari. (2010). Expert systems and decision support systems as input to decision-making in the organization, available on: 
Islamic Economics \& Finance Pedia, accessed online (13-04-2017) at "http://iefpedia.com/arab/wpcontent/uploads/2010/03/"

[9] Bdrany TH., Sailo R. (2014). Evaluation of time series prediction of temperature rates using neural networks. Iraqi Journal of Statistical Sciences, Issue (26), Iraq.

[10] Elglaad, M., A. A. Ewees, E. E. Abd ElRazik, and M. M. Refaat (2016). Computational Comparison of Prediction Algorithms for Predicting Employees Training Needs. IJRIT International Journal of Research in Information Technology, Vol. 4(10), pp. 94-101

[11] Breiman, L. (1996). Bagging predictors. Machine learning 24: 123.

[12] Dimitoglou, G., James, A. A, \& Carol M. J. (2012). Comparison of the C4.5 and a Naive Bayes Classifier for the Prediction of Lung Cancer Survivability. Journal of Computing, Vol. 4 (8).

[13] Seongwook Youn, Dennis McLeod,2007. A Comparative Study for Email Classification, University of Southern California, Los Angeles, CA 90089, USA.

[14] Margaret H. Danham, S. Sridhar, Data mining, Introductory and Advanced Topics, Person education, 1st ed., 2006.

[15] Demuth, H., \& Beale, M. (2009). Matlab neural network toolbox user's guide version 6. The MathWorks Inc.
[16] Rosenblatt, F. (1961). Principles of neurodynamics. perceptrons and the theory of brain mechanisms (No. VG-1196-G-8). Cornell Aeronautical Lab Inc Buffalo Ny.

[17] Rumelhart, D. E., Hinton, G. E., \& Williams, R. J. (1985). Learning internal representations by error propagation (No. ICS-8506). California Univ San Diego La Jolla Inst for Cognitive Science.

[18] Cybenko, G., (1989). Approximation by superpositions of a sigmoidal function._Mathematics of Control, Signals, and Systems, Vol. 2 (4), 303-314 . Springer-Verlag New York Inc.

[19] Nguyen, D., \& Widrow, B. (1990). Improving the learning speed of 2-layer neural networks by choosing initial values of the adaptive weights. In Neural Networks, 1990., 1990 IJCNN International Joint Conference on (pp. 21-26). IEEE.

[20] Chai, Tianfeng, and Roland R. Draxler. Root mean square error (RMSE) or mean absolute error (MAE)? Geoscientific Model Development Discussions 7 (2014): 1525-1534.

[21] Ghose, A., Governatori, G. and Sadnanda, R. (2009). Agent Computing and Multi-Agent Systems: 10th Pacific Rim International conference on Multi-agent PRIMA 2007, Springer village Berlin. 